\title{
13. A MULTIMETHOD ATTRIBUTION ANALYSIS OF THE PROLONGED NORTHEAST BRAZIL HYDROMETEOROLOGICAL DROUGHT (2012-16)
}

\author{
Eduardo S. P. R. Martins, Caio A. S. Coelho, Rein Haarsma, Friederike E. L. Otto, \\ Andrew D. King, Geert Jan van Oldenborgh, Sarah Kew, Sjoukje Philip, \\ francisco C. Vasconcelos Júnior, and Heidi Cullen
}

Northeast Brazil experienced profound water shortages in 2016 due to a five-year drought. Using multiple methods, we could not find sufficient evidence that anthropogenic climate change increased drought risk.

Introduction. The northeast Brazil region (NEB, defined as the land area in $7^{\circ}-21^{\circ} \mathrm{S}, 36^{\circ}-47^{\circ} \mathrm{W}$; Fig. 13.1a) has experienced a remarkable drought during the 5-year period between 2012-16 (Fig. 13.1c). The NEB encompasses the largest regional water supply system of Brazil, the São Francisco River Basin (SFRB), which is of great importance not solely for human consumption, but also for agricultural and hydropower production. During the 2012-16 drought, this system suffered major impacts due to water shortages affecting several sectors. Southern NEB experiences the wet season during austral summer and the dry season during austral winter. Central NEB has a semiarid climate with reduced precipitation, relative to the rest of Brazil, during all seasons. Northern NEB experiences the wet season during austral autumn and is predominantly dry during the other seasons. The region is prone to frequent droughts most often associated with El Niño (Ropelewski and Halpert 1987, 1989) and/or the positive (northward) anomalous sea surface temperature (SST) gradient between tropical north and south Atlantic (Moura and Shukla 1981). However, the beginning of the 2012-16 drought has been documented not to be associated to El Niño (Rodrigues and McPhaden 2014; Marengo et al. 2016).

AFFILIATIONS: MARTINS AND VASCONCELOS JÚNIOR-FUNCEME Research Institute of Meteorology and Water Resources, Fortaleza, Brazil; CoELHO-Center for Weather Forecast and Climate Studies, National Institute for Space Research, Cachoeira Paulista (SP), Brazil; HaARsma, VAN Oldenborgh, KeW, AND PhilipRoyal Netherlands Meteorological Institute, De Bilt, Netherlands; OTTO-Environmental Change Institute, University of Oxford, Oxford, United Kingdom; KING-University of Melbourne, Melbourne, Victoria, Australia; CuLlen-U.S. Climate Central, Princeton, New Jersey

DOI:10.1175/BAMS-D-17-0102.I

A supplement to this article is available online (10.1175 /BAMS-D-17-0102.2)
The SFRB water system (composed of Três Marias, Sobradinho, and Itaparica reservoirs) reached in January 2016 just 5\% of its volume capacity (Fig. 13.1b). Most important reservoirs across other regional states reached similar low levels, causing water shortages in several municipalities. In December 2016, one of the regional states (Ceará), registered 39 collapsed (empty) reservoirs out of 153 monitored reservoirs. Another 42 reached the inactive volume, with waters solely accessible when installing dedicated pumping systems. In addition, 96 out of the 184 Ceará municipalities experienced water supply interruption. To reduce northern basin vulnerability, a long-lasting project dating back to colonial times, was implemented: the São Francisco diversion projecta large-scale interbasin water transfer to the driest NEB portion, bringing southern SFRB water to northern states. Remaining issues to be addressed are the impacts of prolonged droughts on the project sustainability and the potential impact the diversion may have in increasing water demand in the northern basins.

This water crisis is not solely due to the evolving state of the physical system but is also aggravated by various federal and state system structural problems affecting drought monitoring/forecasting, vulnerability assessment, mitigation, and response planning. The crisis is therefore profoundly exacerbated by drought management deficiencies. Both exposure and vulnerability (due to population growth and increased water demand) remain high and can be further intensified with frequent disregard of longterm view in short- and medium-term decisions.

This study investigates possible changes in the hydrometeorological hazard, comprising the accumulated precipitation, the difference between precipitation and evaporation $(P-E)$, and its potential impact on two SFRB reservoirs inflows (Q). A drought as- 
sessment solely based on meteorological aspects is not sufficient to inform public decisions. The combination of the physical event, vulnerability, and exposure of millions of people living in rural and urban areas represent the true impact (Field et al. 2012).

Data and methods. This paper performs an assessment and attribution analysis of the 2016 NEB drought event through a multimethod investigation of 12-month (January to December 2016) and multiyear (2012-16) accumulated precipitation, water balance
$(P-E)$, and 12-month hydrological flow (Q). The methods include:

(i) Estimation of return periods for the 2016 and 2012-16 drought events based on historical records (1900-2016). Return periods were obtained by inverting the fit of annual accumulation of monthly mean precipitation to a Gaussian distribution that scales with the smoothed global mean surface temperature (GMST). Global warming is factored in by allowing the Gaussian fit to be a function of the (low-pass filtered) GMST. It is assumed that the scale parameter (a)

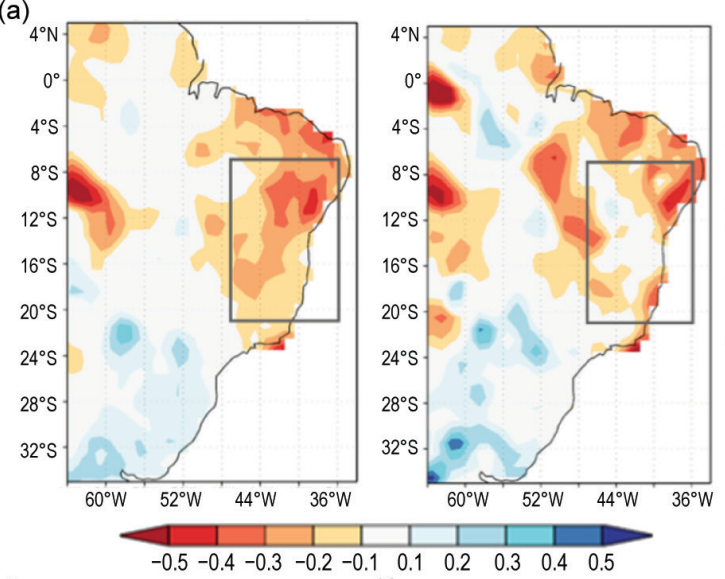

(c)

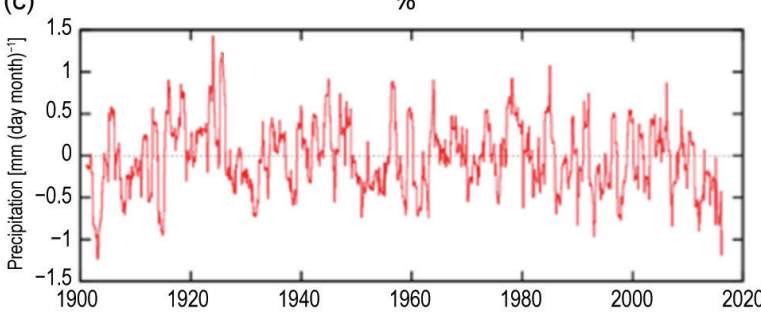

(e)

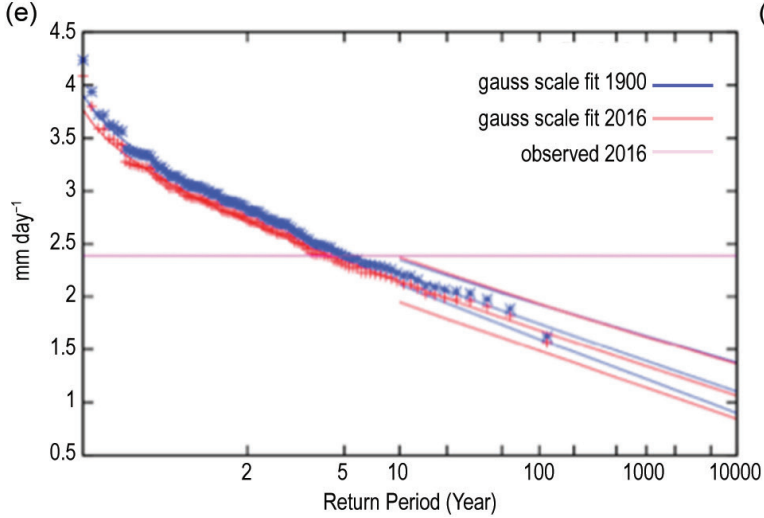

(b)

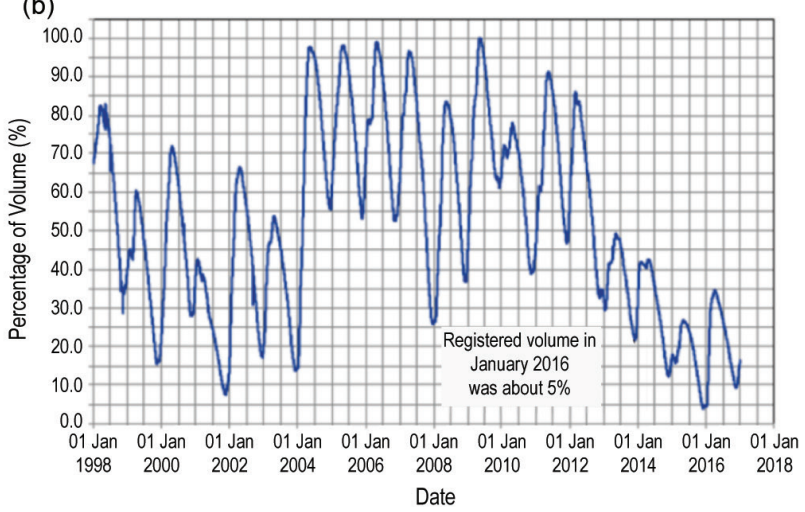

(d)

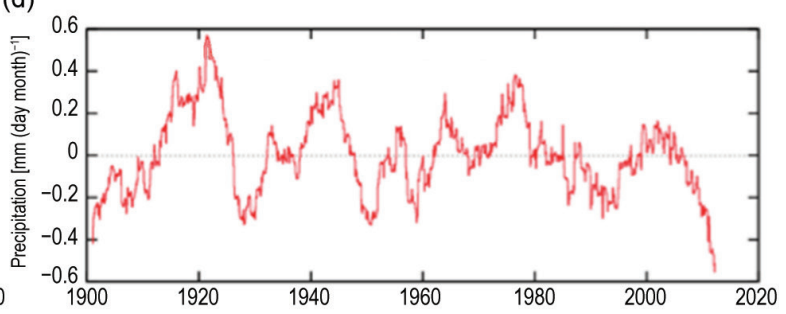

(f)

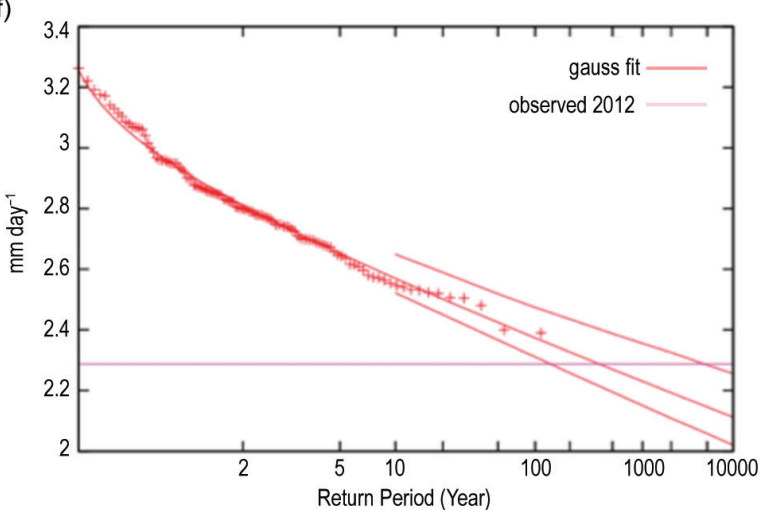

Fig. 13.I. (a) Relative precipitation anomalies for Jan 2012-Dec 2016 (left) and Jan-Dec 2016 (right) as a percentage of the 194I-2010 climatology (Source: GPCC); (b) São Francisco River Basin equivalent reservoir water volume (\%) since 1998; (c) 12-month running mean of precipitation anomalies averaged over land grid points within the area $7^{\circ}-21^{\circ} \mathrm{S}, 36^{\circ}-47^{\circ} \mathrm{E}$. Base period 194I-2010; (d) Same as (c), but for 5 -year running mean; (e) Return period curve obtained by inverting the fit of annual sum of monthly mean precipitation to a Gaussian distribution that scales with the smoothed global mean surface temperature. Observations (pink) are shown twice: scaled to the 2016 climate (red) and to the 1900 climate (blue); (f) As in (e), but now for 5 -year sum and no trend. 
(i.e., the standard deviation) scales with the position parameter (i.e., the mean) of the Gaussian fit. This observational analysis is based on the GPCC-V7 analysis up to 2013 (Global Precipitation Climatology Centre; Schneider et al. 2014), and the GPCC monitoring V5 analysis for 2014-16, designed to be compatible with each other.

(ii) Estimation of the change in drought risk for this event by comparing model simulations of the current climate with simulations of the climate in a "world that might have been" if the atmospheric composition through greenhouse gas emissions had not been changed. We use the distributed computing framework-weather@home-to run the Met Office Hadley Centre atmosphere-only general circulation model HadAM3P (Massey et al. 2015) to simulate precipitation and $P-E$ in two different ensembles representing: 1) observed climate conditions of 2016, and 2) counterfactual conditions under preindustrial greenhouse gas forcings and 11 different SST estimates without human influence (Schaller et al. 2014).

(iii) A similar procedure as in (ii) but instead using coupled multimodel ensemble simulations (CMIP5; Taylor et al. 2012) and the SST-forced HadGEM3-A model (Christidis et al. 2013).

(iv) Downscaling HadAM3P precipitation and evaporation using a hydrological model (Lopes et al. 1981) for estimating flows for both High (Três Marias) and Medium (Sobradinho) São Francisco hydrographic regions.

Results. Drought conditions were observed over NEB during 2012-15 and continued into 2016 for most of the region (Fig. 13.1a). Figures 13.1c,d show NEB 12-month and 5-year running mean time series, respectively. While the severity of the 2012-16 drought is evident, no historical trend is discernible in either of the series. The return period for the 2016 drought is about 4 years [ $95 \%$ confidence interval (CI): $2-9$ years (Fig. 13.1e)]; however the continuous 2012-16 drought has a return period of 350 years [95\% CI: at least 135 years (Fig. 13.1f)], characterizing this drought as exceptional. There is no autocorrelation in the series, so the 5-year drought is a combination of 1-year droughts. Note that with 100 years of this data, only trend changes that exceed a roughly twofold increase or decrease in probability can be detected.

The NEB annual mean precipitation weather@ home analysis (Fig. 13.2a) shows that low precipitation extremes have become slightly less likely due to anthropogenic forcing: what would have been a 1-in-4-year precipitation deficit event like the 2016 event has become approximately a 1-in-6-year event with a risk ratio of 0.70 (95\% CI: $0.55-0.84)$. The $P-E$ analysis (Fig. 13.2d) also indicates a reduction in drought risk. For future precipitation projections under a $2^{\circ} \mathrm{C}$ scenario (Mitchell et al. 2017), the picture is different (not shown) with a marked increase in low precipitation extremes in consistency with the CMIP5 analysis below.

Our CMIP5 analysis used eight climate models passing our evaluation test of satisfactorily capturing the observed NEB annual precipitation anomalies distribution (see online supplementary material). Using these models we compared the likelihood of 1- and 5-year precipitation deficits comparable to the 2016 and 2012-16 events, respectively (Figs. 13.2b,e). Our multimodel analysis indicates that climate change has increased the probability of such prolonged low precipitation events, although there is high uncertainty on the magnitude of that influence (Fig. 13.2i). In future, precipitation deficits like 2016 or the last five years are projected to be even more likely. There is also no detectable change in $P-E$ due to human-induced climate change (Fig. 13.2c,f) presumably because the increase in evaporation cancels the increase in precipitation. The HadGEM3-A analysis indicates reduced risk for low precipitation events due to anthropogenic forcing, with even higher uncertainty than CMIP5 (Figs. 13.2i).

The comparison of the probability density functions (PDF) for 2016 annual flow under preanthropogenic (counterfactual ensemble) and current emissions (actual ensemble) for both SFRB regions (Figs. 13.2g,h) reveals slightly reduced risk of extreme low flow as observed in 2016 due to anthropogenic forcing.

Conclusions. The observational analysis confirmed that droughts are common over NEB, but prolonged droughts comparable to the current one are exceptional, as highlighted by the impressive return period for the 2012-16 drought of at least 135 years.

Theweather@home simulations indicated that anthropogenic climate change is not contributing to increased risk of single-year droughts over NEB, which is in line with the hydrological analysis that also did not indicate increased risk for extreme low flow. This is consistent with the observational analysis that did not indicate a trend toward drier conditions up to now as an association with global mean temperature (see Fig. ES13.1e). Despite the CMIP5 analysis indicating increased likelihood of 1- and 5-year precipitation deficits over NEB due 
to anthropogenic forcing, an uncertainty analysis of the 1-year precipitation risk ratio results shows that the evidence is weighted toward natural climate variability as the principle driver, as summarized in Fig. 13.2i. Most CIs include the risk ratio equal to 1 indicating that no change in drought risk can be detected or attributed. Our multimethod analysis suggests that there is not enough evidence that anthropogenic climate change increased drought risk. In future projections under strong radiative forcing, bothweather@home and CMIP5 indicate increased risk for extremely dry events.

(a)
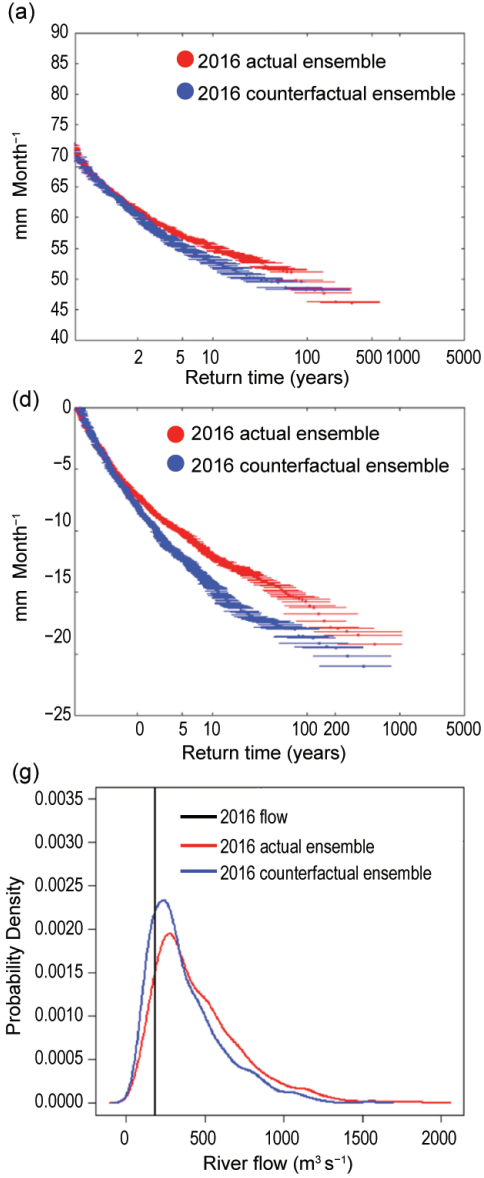

(b)

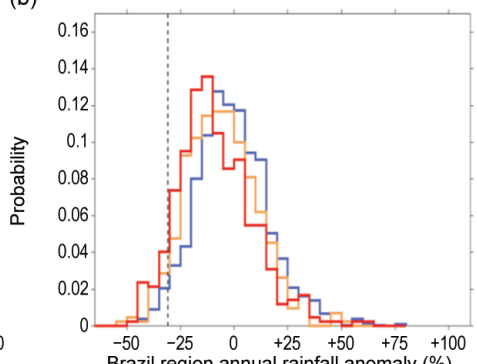

(e)

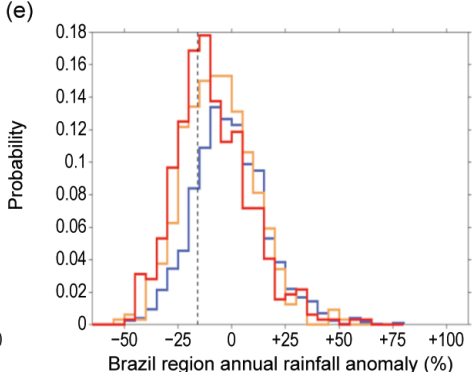

(h)

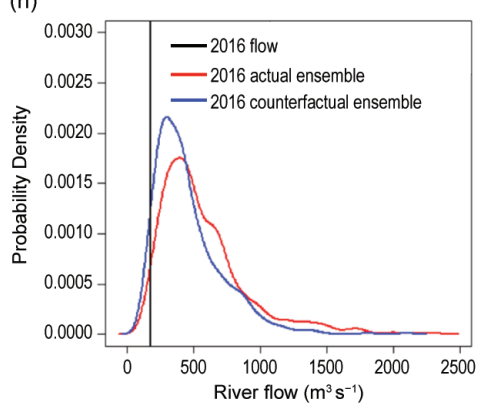

The 2012-16 drought might also have been prolonged by a positive hydrological cycle feedback. The possibility of a positive feedback between precipitation and soil moisture and the existence of multiple equilibria was theoretically suggested by D'Andrea et al. (2006). Oyama and Nobre (2003) and Hirota et al. (2011) investigated this feedback for NEB showing that land surface and vegetation changes could induce tipping points and multiple equilibria. A similar investigation focused on the 2012-16 event could help advance the understanding of the mechanisms associated to the observed drought.

(c)

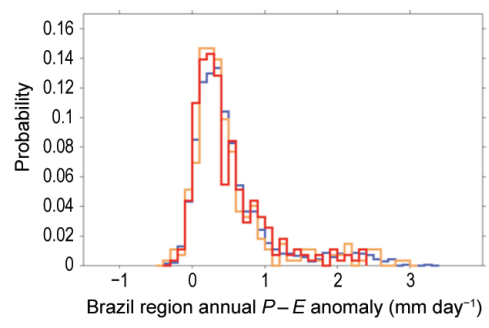

(f)

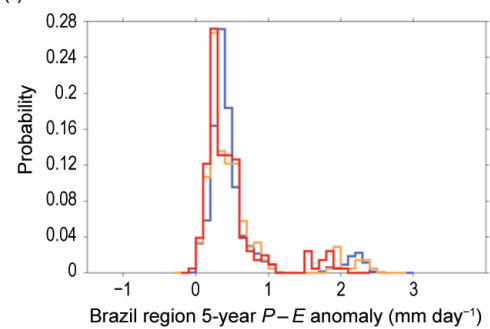

(i)

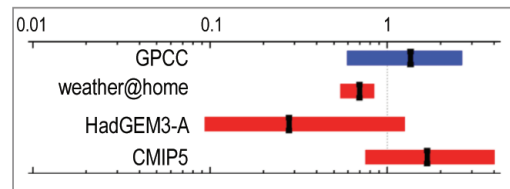

FIG. 13.2. (a) Return period curve obtained by inverting the empirical distribution fit of total precipitation averaged over NEB land grid points for the year 2016 in HadAM3P; (b) PDF of annual precipitation anomalies (from a 1961-90 historical climatology) averaged over NEB land grid points in climate simulations under natural influences only (blue), all-forcings (orange) and projected forcings under the RCP8.5 scenario in 2050 (red). The dashed line shows the observed 2016 anomaly; (c) Same as (b), but for annual P-E anomalies; (d) Return period curve of I2-month mean P-E (for the year 2016) averaged over NEB land grid points in HadAM3P; (e) Same as (b), but for 5-year precipitation anomalies. The dashed line shows the observed 20I2-16 anomaly; (f) Same as (e), but for 5-year P-E anomalies; (g) High São Francisco (Três Marias); (h) Medium São Francisco (Sobradinho) annual flow PDF for 2016 estimated using HadAM3P simulations; (i) Risk ratio and 95\% Cls (represented by the horizontal thick bars) for annual precipitation accumulation in GPCC, weather@home, HadGEM3-A, and CMIP5. HadGEM3-A experiments were performed for the European Climate Extremes: Interpretation and Attribution (EUCLEIA) project by the Met Office. A risk ratio larger (smaller) than I indicates a trend toward more (less) severe droughts. 
Government responses to the past and present droughts have common characteristics that severely prevent drought risk mitigation through improved response and relief, long-term resilience building, and adaptation measures (Martins et al. 2016). This is particularly true for the multiyear drought (2012-16) analyzed in this paper.

ACKNOWLEDGMENTS. We thank our colleagues at the Oxford eResearch Centre, A. Bowery, M. Rashid, S. Sparrow, and D. Wallom and the Met Office Hadley Centre PRECIS team for its technical and scientific support for the development and application of weather@home. We thank CNPq (processes 304586/2016-1 and 309499/2013-5) and FAPESP (CLIMAX project, 2015/50687-8) for supporting the development of this study and the funders of the World Weather Attribution program.

\section{REFERENCES}

Christidis, N., P. A. Stott, A. A. Scaife, A. Arribas, G. S. Jones, D. Copsey, J. R. Knight, and W. J. Tennant, 2013: A new HadGEM3-A-based system for attribution of weather- and climate-related extreme events. J. Climate, 9, 2756-2783, doi:10.1175/JCLI-D-12-00169.1.

D'Andrea, F., A. Provenzale, R. Vautard, and N. De Noblet-Decoudré, 2006: Hot and cool summers: Multiple equilibria of the continental water cycle. Geophys. Res. Lett., 33, L24807, doi:10.1029/2006GL027972.

Field, C. B., and Coauthors, 2012: Managing the Risks of Extreme Events and Disasters to Advance Climate Change Adaptation. Cambridge University Press, 582 pp.

Hirota, M., M. Holmgren, E. H. Van Nes, and M. Scheffer, 2011: Global resilience of tropical forest and savanna to critical transitions. Science, 334, 232-235, doi:10.1126 /science.1210657.

Lopes, J. E. G., B. P. F. Braga Jr., and J. G. L. Conejo, 1981: Hydrological simulation: Application of a simplified model (in Portuguese). Annals of III Brazilian Symposium on Water Resources (Fortaleza)/ABRH, 2, 42-62.

Marengo, J. A., R. R. Torres, and L. M. Alves, 2016: Drought in Northeast Brazil-past, present, and future. Theor. Appl. Climatol., 129, 1189-1200 doi:10.1007/ s00704-016-1840-8.

Martins, E. S. P. R., F. J. C. Teixeira, J. G. L. Conejo, J. Machado, and A. D. Moura, 2016: Crisis, opportunity, and leadership. Drought in Brazil: Proactive Management and Policy, E. De Nys et al. Eds., CRC Press, 19-26.
Massey, N., and Coauthors, 2015: weather@home-development and validation of a very large ensemble modelling system for probabilistic event attribution. Quart. J. Roy. Meteor. Soc., 141, 1528-1545, doi:10.1002/q j.2455.

Mitchell, D., and Coauthors, 2017: Half a degree additional warming, projections, prognosis and impacts (HAPPI): Background and experimental design. Geosci. Model Dev., 10, 571-583, doi:10.5194/gmd10-571-2017.

Moura, A. D., and J. Shukla, 1981: On the dynamics of droughts in Northeast Brazil: Observations, theory, and numerical experiments with a general circulation model. J. Atmos. Sci., 38, 2653-2675, doi:10.1175/1520-0469(1981)038<2653 :OTDODI>2.0.CO;2.

Oyama, M. D., and C. A. Nobre, 2003: A new climate-vegetation equilibrium state for tropical South America. Geophys. Res. Lett., 30, 2199, doi:10.1029 /2003GL018600.

Rodrigues, R. R., and M. J. McPhaden, 2014: Why did the 2011-2012 La Niña cause a severe drought in the Brazilian Northeast? Geophys. Res. Lett., 41, 1012-1018, doi:10.1002 /2013GL058703.

Ropelewski, C. F., and M. S. Halpert, 1987: Global and regional scale precipitation patterns associated with the El Niño/Southern Oscillation. Mon. Wea. Rev., 115, 1606-1626, doi:10.1175/1520-0493(1987)115<1606:GARSPP>2.0.CO;2.

$\longrightarrow$, and — 1989: Precipitation patterns associated with the high index phase of the Southern Oscillation. J. Climate, 2, 268-284, doi:10.1175/1520-0442(1989)002<0268 :PPAWTH>2.0.CO;2.

Schaller, N., J. Sedláček, and R. Knutti, 2014: The asymmetry of the climate system's response to solar forcing changes and its implications for geoengineering scenarios. J. Geophys. Res. Atmos., 119, 5171-5184, doi:10.1002/2013JD021258.

Schneider, U., A. Becker, P. Finger, A. Meyer-Christoffer, M. Ziese, and B. Rudolf, 2014: GPCC's new land surface precipitation climatology based on quality-controlled in situ data and its role in quantifying the global water cycle. Theor. Appl. Climatol., 115, 15-40, doi:10.1007/s00704 -013-0860-x.

Taylor, K. E., R. J. Stouffer, and G. A. Meehl, 2012: An overview of CMIP5 and the experiment design. Bull. Amer. Meteor. Soc., 93, 485-498, doi:10.1175/BAMS-D-11-00094.1. 